\title{
Slope stability analysis in the case of probabilistic and semi-probabilistic design method
}

\author{
Hubert Szabowicz* \\ Wrocław University of Science and Technology, Faculty of Civil Engineering, Wyb. Wyspiańskiego \\ 27, 50-370 Wrocław, Poland
}

\begin{abstract}
This paper addresses the issue of probabilistic and semiprobabilistic modelling of soil slopes. A slope made of cohesive-frictional soil of specific geometry was analysed as an example. Results were calculated for two methods using the Z-Soil finite element software. It has been assumed that the probability distributions of strength parameters, cohesion and internal friction angle are normal distributions with average values and coefficient of variation $=0.2$. Random finite element method (RFEM) has been used for probabilistic modelling. Random fields of cohesion and internal friction angle have been generated using the Fourier series method (FSM). Monte Carlo simulation has been used to calculate the statistics of the slope factor of safety in order to determine the probability of failure. Moreover, assumed parameter distributions allowed to determine safe characteristic values used in the semi-probabilistic partial factors method. Both approaches have been compared in the article.
\end{abstract}

\section{Introduction}

Slopes are the most basic and common elements of geotechnical structures. They are used during trench excavation for large-size structures when the construction site area does not require using protective elements such as retaining walls and allows for sloping instead, in linear structures such as roads and railroads located on embankments or in wide excavations, and at dumping grounds, waste dumps, floodbanks, etc.

The design engineer may be tasked with designing a completely new structure as well as evaluating the state of an existing one. It is often necessary to evaluate the causes of failures, damage, and landslides.

The crucial aspect during the process of designing aforementioned structures is the verification of their overall stability. According to Eurocode [1], GEO and STR ultimate limit states are to be checked in order to analyse the stability of geotechnical structures.

No analytical solution to the problem of stability of geotechnical structures has been developed so far. Multiple methods are available in order to provide an approximate evaluation of the safe design of a structure. Since the first half of the 20th century slope stability was analysed by means of limit equilibrium methods (Fellenius, Bishop) where the

\footnotetext{
* Corresponding author: hubert.szabowicz@gmail.com
} 
stability was determined by a value calculated as a ratio of forces stabilizing the slope to destabilizing ones. These methods allowed to determine the critical slip line (it was assumed that the failure occurs on the line with the lowest safety factor). Because of strong simplification (including shapes of allowed mechanism and agreements of soil layers) needed to be used for these methods, they are currently consider as only approximates ones and their practical use requires assuming high safety factors [2].

The development of numerical methods enabled triggered their use to analyse factor of safety. Currently the shear strength reduction (SSR) method is the most common method used to determine slope stability. In contrary to the earlier methods, this method provide considerably more precise results due to the possibility of accounting for other hydrogeological processes occurring in the substrate and much more precise modelling of failure mechanisms.

Standards and regulations applicable in Poland allow for calculation of structures using semi-probabilistic methods using partial factors (the most common approach) as well as probabilistic methods taking into consideration actual probability distribution of parameter. Probabilistic methods are increasingly common due to the development and availability of numerical software. Random finite element method (RFEM), developed in the framework of the random field theory, is an increasingly common approach. The method in general consist of analysing series of Monte Carlo simulations, where in each simulation spatial variability of parameter is presented in the form of random field. The method was proposed in the pioneer papers in the 1990s and quickly became applied in the slope analysis process [3]. Currently they are widely used (however, rather rarely in practical engineering) for purposes such as determining safety of shallow foundations [4], supported excavation [5] or pile foundation [6].

\section{Semi-probabilistic modelling of slopes - numerical methods}

Overall stability analysis has been performed using ZSoil finite element code. Safety factor was determined using the shear strength reduction (SSR) method mentioned above [7]. Soil material has been described using the Coulomb-Mohr elastic-perfectly plastic model with non-associated flow rule (dilatancy angle $\psi=0$ ).

The shear strength reduction method includes simultaneous reduction of the internal friction angle $\phi$ and cohesion $c$ until a state of shearing failure on the slip surface is achieved according to the following formula (1) [8]:

$$
\int_{\Gamma_{S}} \tau d \Gamma_{S}=\frac{\int_{\Gamma_{S}} \tau_{y} d \Gamma_{S}}{S F}=\frac{\int_{\Gamma_{S}}\left(c+\sigma_{n} \tan \phi\right) d \Gamma_{s}}{S F}
$$

where:

$\tau$ - shear stress,

$\tau_{y}$ - yield stress according to Coulomb-Mohr criterion,

$\sigma_{n}-$ effective normal stress,

$S F$ - stability - factor for which instability was achieved,

$\Gamma_{S}$ - sliding surface.

The algorithm for calculating the safety factor is as follows:

- initialisation - answering the problem for the initial factor $S F_{0}=1$,

- increasing factor $S F_{n}$ gradually to decrease $c$ and $\tan \phi$ parameters in successive iterations, 
- when convergence of calculations is not obtained, the factor is reduced by a half of the first step value (increasing the precision of calculations),

- iterations are repeated until satisfactory precision is achieved.

Failing to acquire convergence is related to the considerable strain occurring in the failure area (along the slip surface) and it means that the structure begins to lose stability. The development of shearing strain for various locations of the slip surface are presented below in section 6

According to the semi-probabilistic method, the structure is safe when it meets the condition described by the following formula (2):

$$
S F>S F_{\text {lim }}
$$

where the limit of the stability index $S F_{\text {lim }}=1,0$.

Eurocode [1] defines four possible calculation approaches for the semi-probabilistic method. They differ by the method of assuming the values of individual partial factors divided into three groups:

$-A-$ used for actions and their effects

$-M-$ used for soil parameters,

$-R$ - used for resistive force on the sliding surface

The values of partial factors are presented in Table 1.

Table 1. Partial factors for slope stability analysis [1]

\begin{tabular}{|c|c|c|c|c|c|c|}
\hline \multirow{3}{*}{\multicolumn{2}{|c|}{ Partial factors }} & \multicolumn{4}{|c|}{ Approach to calculation } & \multirow{4}{*}{$\begin{array}{c}\text { Partial factors for: } \\
\text { Permanent adverse actions }\end{array}$} \\
\hline & & \multicolumn{2}{|c|}{1} & \multirow{3}{*}{$\begin{array}{c}\mathbf{2} \\
\text { (DA2) } \\
1.35 \\
\end{array}$} & \multirow{3}{*}{$\begin{array}{c}\mathbf{3} \\
(\mathbf{D A 3}) \\
1.0 \\
\end{array}$} & \\
\hline & & (DA1-1) & (DA1-2) & & & \\
\hline \multirow{3}{*}{$A$} & $\gamma_{G}$ & 1.35 & 1.0 & & & \\
\hline & $\gamma_{G f a v}$ & 1.0 & 1.0 & 1.0 & 1.0 & Permanent favourable actions \\
\hline & $\gamma_{Q}$ & 1.5 & 1.3 & 1.5 & 1.3 & Variable actions \\
\hline \multirow{3}{*}{$M$} & $\gamma_{\phi}$ & 1.0 & 1.25 & 1.0 & 1.25 & Shearing resistance \\
\hline & $\gamma_{c}$ & 1.0 & 1.25 & 1.0 & 1.25 & Effective cohesion \\
\hline & $\gamma_{\gamma}$ & 1.0 & 1.0 & 1.0 & 1.0 & Weight density \\
\hline$R$ & $\gamma_{R ; e}$ & 1.0 & 1.25 & 1.1 & 1.0 & $\begin{array}{c}\text { Shearing resistance on slip } \\
\text { surface }\end{array}$ \\
\hline
\end{tabular}

It must be noticed that approaches DA1-2 and DA2 may cause complications when used for numerical methods due to the difference between the partial safety factor values for stabilizing and destabilizing forces. Guidelines for using these values for numerical methods were presented in paper [9]. For example, for the DA1-1 approach, the unit weight of soil should be multiplying by factor $\gamma_{G}=1,3$, the variable load by $\gamma_{Q}=1,5$. The other factors and the limit value $S F$ should be unchanged.

According to paper [10], DA1-1 approach should be used when the characteristic values of strength parameters of soil have been determined precisely, ensuring a high degree of recognition of geotechnical conditions of the whole soil mass. In this paper it has been assumed that the probability distribution of strength parameters are known, it may be thus presupposed that the condition of precise determination of strength parameters is satisfied. Therefore, this approach has been used in calculations after implementing said modification. 


\section{Probabilistic modelling of slopes}

The Eurocode [11] allows for analysing safety factor using a probabilistic method that enables precise determination of the probability of a failure. It is described as the probability of safety factor being smaller than 1.0 and expressed with the following formula (3) $[12]$ :

$$
p_{f}=P\left(S F\left(x_{1}, x_{2}, \ldots, x_{n}\right)<1.0\right)
$$

where $x_{1}, x_{2}, \ldots, x_{n}$ are random variables determining the function estimating the slope stability.

For a structure of RC2 reliability class (average threat to human life or major economic, social, and environmental consequences) and assumed 50-year lifetime, the limit of the probability of failure $p_{f}=7 \cdot 10^{-5}$.

Probabilistic modelling consists mainly on replacing deterministic parameter values of a problem with its probability distributions. Therefore, it is not necessary to determine factors such as safe characteristic values. Instead, the probability distribution of a given parameter is determined and implemented to the task as a whole. In the case of the Random Finite Element Method (RFEM) [13] used here, the strength characteristics of soil are expressed with random fields. The variability of loads and own weight of the ground has been taken into consideration by using partial factors analogous to the factors applied in the semiprobabilistic method.

To define the random field, apart of point statistics scale of fluctuation (SOF) need to be defined. This scale is a convenient measure of the distance within which point values are significantly correlated [14]. As a rule, in the case of a soil mass the vertical fluctuation scale is considerably smaller than the horizontal scale. This reflects the layered structure of the medium.

Numerous methods are available to generate random fields of a given distribution and of a vertical or horizontal fluctuation scale. The Fourier Series Method (FSM) [15] is an example of such a method.

For typical fluctuation scale values, i.e. horizontal $=10 \mathrm{~m}$ and vertical $=1 \mathrm{~m}$, an example realization of a field generated using this method for given geometry is presented in Fig. 1.

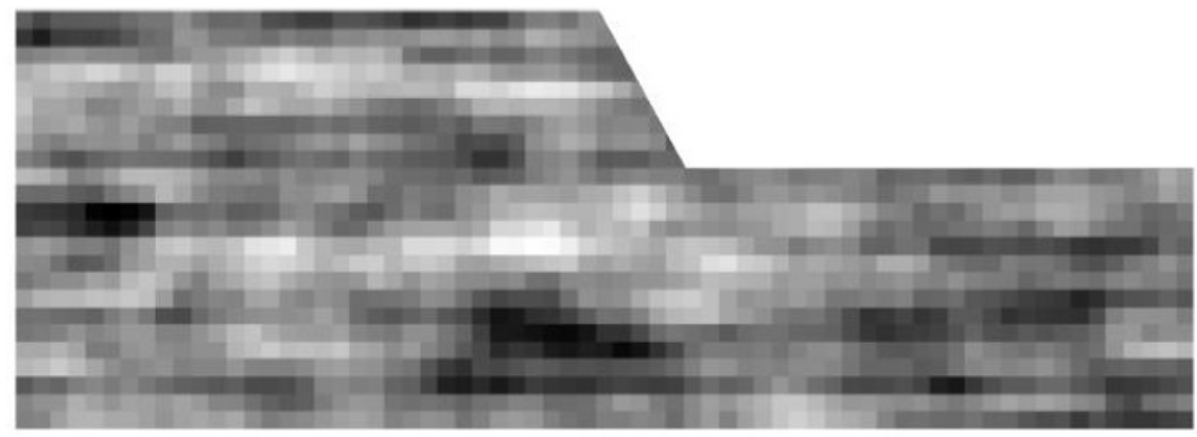




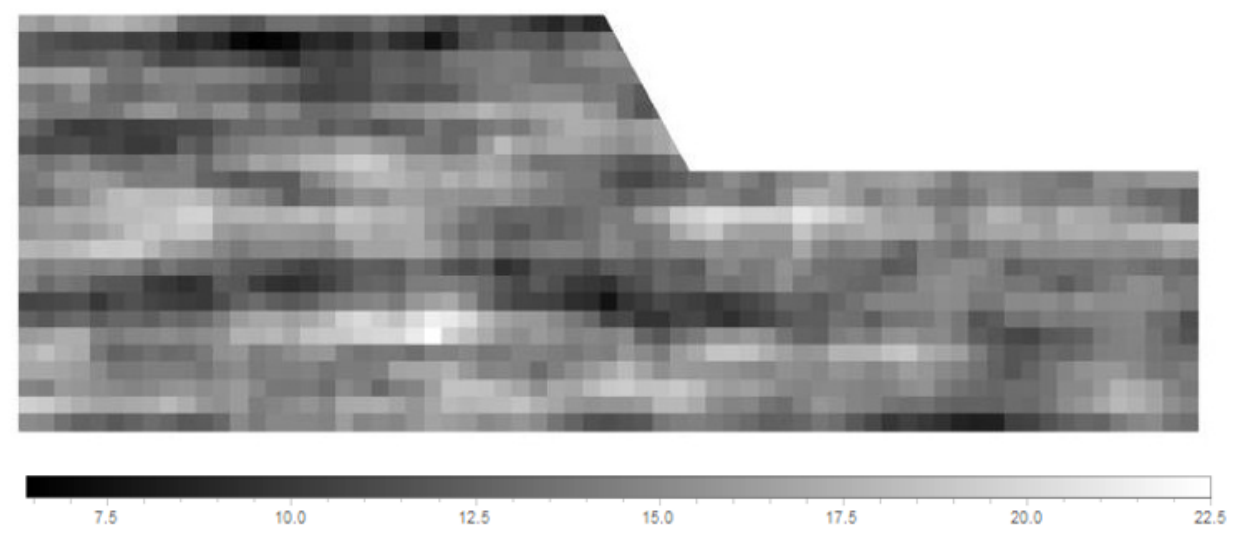

Fig. 1. Single representation of a cohesion (top) and internal friction angle (bottom) field

\section{Numerical example}

In this paper, an attempt was made to solve the problem of the stability of a slope of given geometry using two methods, i.e. probabilistic and semi-probabilistic. In both cases, the problem was solved using the finite element method in Z-Soil environment. All calculations were done using a model of a regular square grid of $0,5 \mathrm{~m}$. The surface of the slope has been modelled using quadrangular elements and triangles created by trimming the square elements. The grid is presented in Fig. 2.

Due to a very minor influence of the soil elastic parameters of $S F$ as shown in paper [16], typical, deterministic values of Young's modulus $E=1000 \mathrm{MPa}$ and Poisson ratio $v=0.3$. were assumed.

The following parameters were used in calculation:

- surcharge characteristic load $q_{k}=4,0 \mathrm{kPa}$, design value $q_{d}=1.5 \cdot q_{k}=6,0 \mathrm{kPa}$,

- characteristic ground weight $\gamma_{k}=20,0 \frac{\mathrm{kN}}{\mathrm{m}^{3}}$, design value $\gamma_{d}=1.3 \cdot \gamma_{k}=26,0 \frac{\mathrm{kN}}{\mathrm{m}^{3}}$.

Random parameters of cohesion and internal friction angle are presented in Table 2.

Table 2. Strength parameters of ground area

\begin{tabular}{|c|c|c|c|}
\hline Effective parameter & Average & Standard deviation & Quantile 5\% \\
\hline Cohesion & $\bar{c}=25,00 \mathrm{kPa}$ & $\sigma_{c}=4,224 \mathrm{kPa}$ & $c_{0,05}=18,05 \mathrm{kPa}$ \\
\hline Internal friction angle & $\bar{\phi}=15,00^{\circ}$ & $\sigma_{f}=2,527^{\circ}$ & $f_{0,05}=10,85^{\circ}$ \\
\hline
\end{tabular}

As it has already been mentioned, the problem was solved using two methods. In the case of probabilistic modelling, calculations were done for 1000 realizations of random fields of $c$ and $\phi$. The statistics of obtained results were used to determine the probability of a failure. The results are presented in section 5For the semi-probabilistic method only a single calculation for design values of parameters and loads obtained from partial factors described in Table 1 was performed. The knowledge of probability distribution was applied to determine a safe evaluation of characteristic values of strength parameters. 


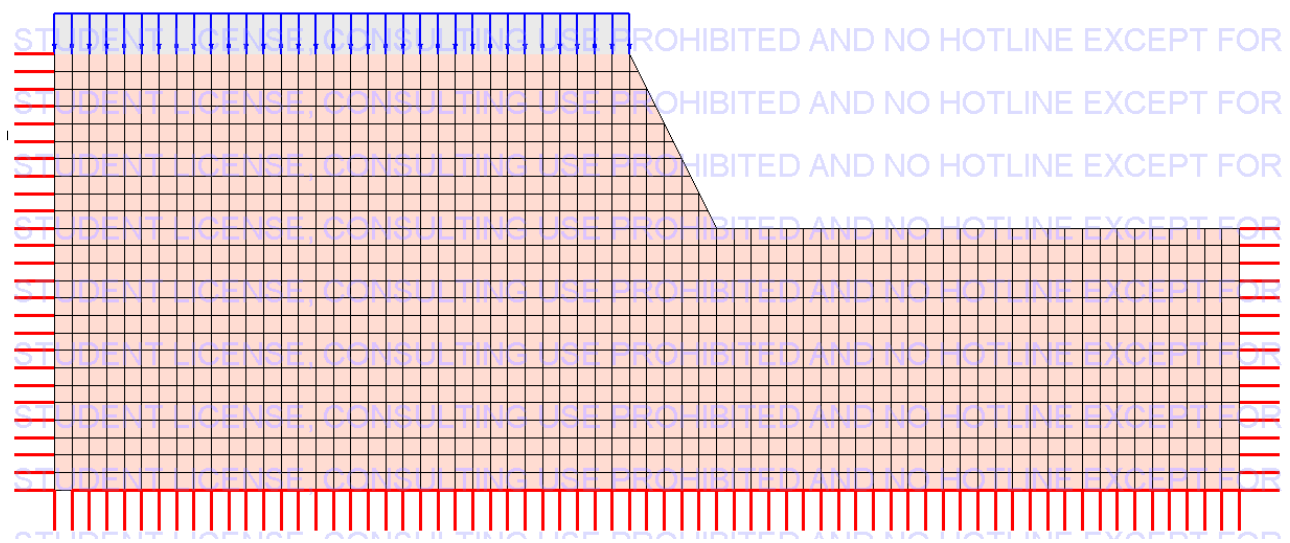

Fig. 2. Computational model

\section{Probabilistic method - result of calculations}

As a result of calculations, 1000 safety factors have been obtained. The results are shown in the histogram in Fig. 3.

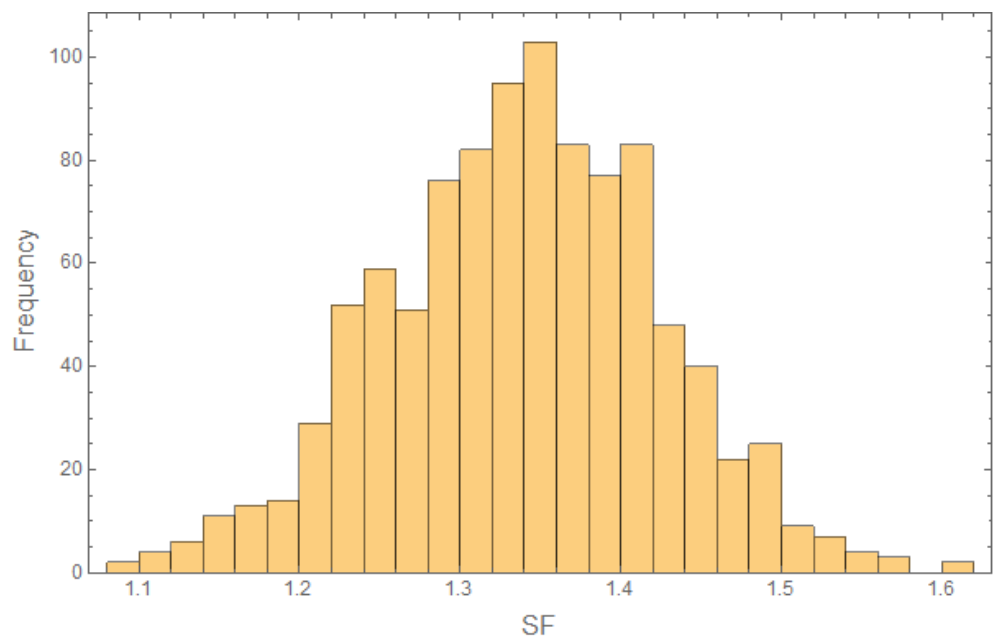

Fig. 3. SF values - histogram

Based on the shape of the histogram, it has been deduced that the safety factor distribution can be described using normal distribution. The following parameters were calculated:

- expected value $\overline{S F}=1.3388$

- standard deviation $\sigma_{S F}=0.08546$

Fig. 4. shows the estimated function of probability distribution described by these parameters (blue) and a smoothed histogram of results (orange). 


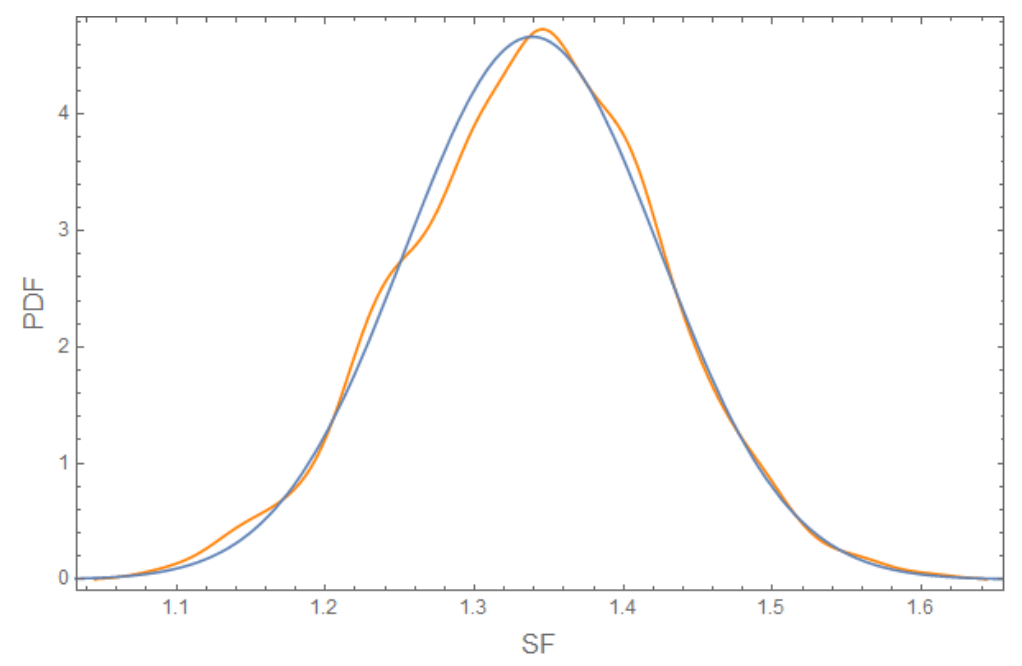

Fig. 4. Safety factor probability density distribution

Clearly, the assumed distribution describes the obtained results rather well. In order to verify if the population distribution $S F$ differs from the assumed normal distribution, the Kolmogorov-Smirnov test has been done. For estimated parameters of the normal distribution, the test result was $48 \%$, which is a satisfactory result for 1000 representations. There is no reason to reject a hypothesis that the obtained sample follows the estimated distribution.

Subsequently, the probability of a failure was determined according to formula (2), in order to check if it meets the requirements of Eurocode [11] for RC2 structures and assumed 50-year service life (admissible probability of failure $p_{f, \text { lim }}=7 \cdot 10^{-5}$ ).

$$
p_{f}=P(S F(c, \phi)<1.0)=3,68 \cdot 10^{-5}<p_{f, l i m}
$$

It may be stated that based on the results of a probabilistic method the slope is designed correctly and meets the regulatory requirements.

Calculating the $7 \cdot 10^{-5}$-quantile of the estimated normal distribution function, $S F_{7 \cdot 10^{-5}}=1,0134$ factor value is obtained. It can be compared with the value obtained using the semi-probabilistic method. Based on the margin of safety, it may be stated that even with slightly lower average values of parameters the value of probability of failure would still not be exceeded.

\section{Semi-probabilistic method - result of calculations}

The semi-probabilistic method includes taking into consideration the randomness of parameters by assuming the characteristic value as a $5 \%$-quantile (presented in Table 2.) and acquiring a design value dividing it by an appropriate partial safety factor $\left(\gamma_{c}=\gamma_{\phi}=\right.$ $1,0)$. For these and presented in section 4values one calculation was carried out.

Calculations done with the assumption of a homogeneous ground area of given parameters have shown that the safety factor $S F=0.9728$, which is below the admissible $S F_{\text {lim }}=1.0$. This means that a slope of assumed geometry in given conditions fails to meet the safety requirements and thus requires redesigning by means such as reducing the slope angle.

The development of shearing strain zones for raising safety factor is shown below. 

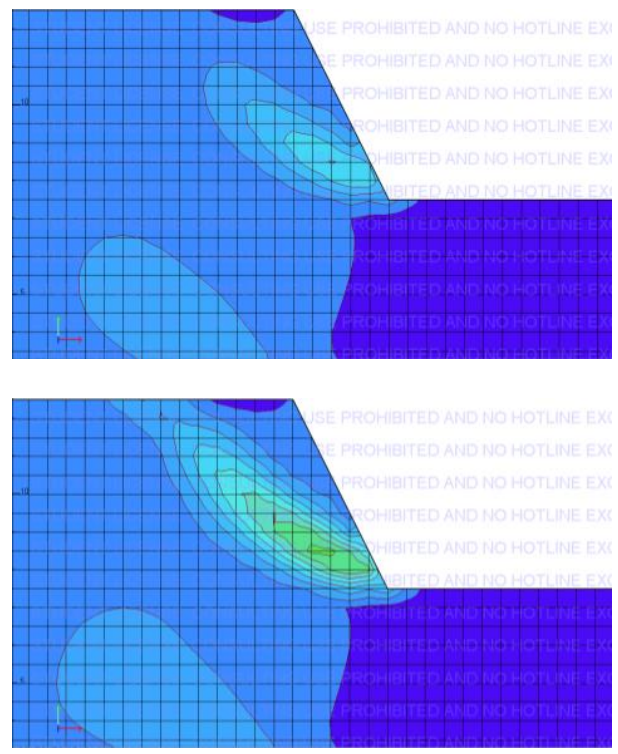
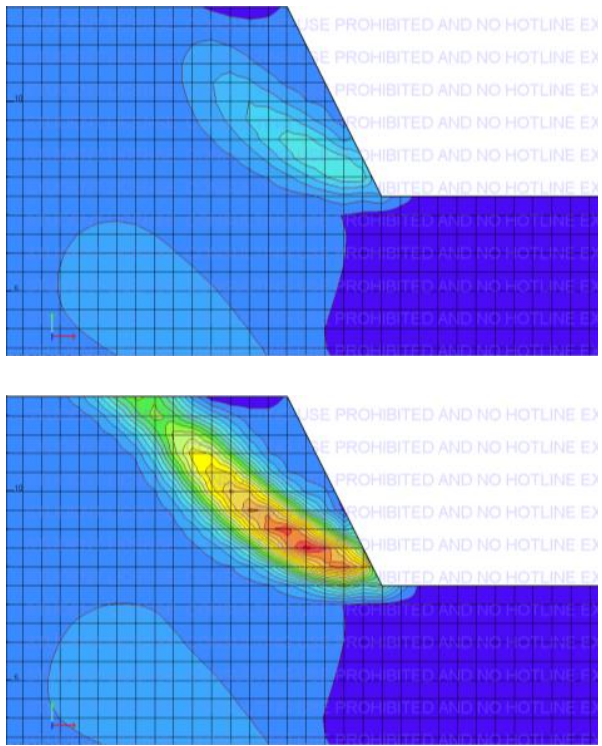

Fig. 5. The development of non-dilatational strain area for subsequent SF values: 0.94, 0.96, 0.97, 0.9728

\section{Summary and conclusions}

Based on the calculations presented above, the application of the semi-probabilistic method with partial factors is conservative and leads to providing wider margin of safety. It does not enable precise determination of the safety of a structure from the probabilistic perspective. It uses factors based on general experience that do not necessarily reflect actual conditions of a given structure. In this case this method resulted in a $4 \%$ margin of safety.

In order to ensure more optimal designing it is recommended to do a more exhaustive analysis of the substrate and determine its random characteristics. Such an approach allows for a precise evaluation of the safety of a structure. Moreover, it enables more precise determination of sliding zones taking into consideration the effects of local weakness or interbedding, allowing us to identify a possible failure area that requires strengthening. It is a good idea to conduct observation-based model inspection and calibration throughout project implementation in order to improve safety even further [17].

It is also of significant importance to select appropriate tools to generate random fields for subsequent calculations and doing a sufficient number of calculations to estimate the results based on the assumed distribution with adequate accuracy. It must be remembered that the accuracy of estimated structure safety depends on the tools and precision.

It is difficult to generalise obtained results as they apply only for a given slope geometry. However, it does not hinder the validity of probabilistic methods. In fact, it is the opposite - even if the observed dependence is not general and the semi-probabilistic method is not always conservative, the application of probabilistic methods enables a reliable determination of the safety of the designed structure.

The author would like to thank Marek Kawa for his assistance in preparing numerical model and for all his factual remarks. 


\section{References and materials consulted}

1. EN 1997, Geotechnical design - Part 1: General rules

2. M. Cała, J. Flisiak, A. Tajduś, Numerical methods for analysis of slope and escarpment stability, Warsztaty z cyklu "Zagrożenia naturalne w górnictwie". Bełchatów, Wydaw. IGSMiE PAN, 2-4 czerwca 2004

3. D.V. Griffiths, J. Huang, G.A. Fenton, Influence of spatial variability on slope reliability using 2-D random fields, Journal of Geotechnical and Geoenvironmental Engineering, 135(10), 1367-1378 (2009)

4. M. Kawa, W. Puła, M. Suska, Random analysis of bearing capacity of square footing using the LAS procedure, Studia Geotechnica et Mechanica, 38(3), 3-13 (2016)

5. M. Kawa, I. Bagińska, M. Wyjadłowski, Reliability analysis of sheet pile wall in spatially variable soil including CPTu test results, Archives of Civil and Mechanical Engineering, 19(2), 598-613, (2019)

6. W. Puła, J. Rybak J, Case history: Pile foundations of a large gantry crane, CISM International Centre for Mechanical Sciences, Courses and Lectures, 491, 185-199 (2007)

7. E.M. Dawson, W.H. Roth, A. Drescher, Slope stability analysis by strength reduction, Geotechnique, 49(6), 835-840 (1999)

8. ZSOIL.PC 2016 User Manual. Theory, Zace Services Ltd, Software engineering, Switzerland

9. A. Batog, E. Stilger-Szydło, Stability of slopes in the case of road embankmentsfrom the perspective of Eurocode 7, Drogownictwo 1/2010

10. A. Batog, E. Stilger-Szydło, Slope stability of embankments of modernised S-8 major road from the perspective of Eurocode 7 and current national regulations, Drogownictwo 2/2010

11. EN 1990, Basis of structural design

12. G. Vessia, J. Kozubal, W. Puła, High dimensional model representation for reliability analyses of complex rock-soil slope stability, Archives of Civil and Mechanical Engineering, 17(4), 954-963 (2017)

13. D.V. Griffiths, G.A. Fenton, Probabilistic slope stability analysis by finite elements, J.Geotech. Geoenviron. Eng., 130(5), 507-518 (2004)

14. I. Bagińska, M. Kawa, W. Janecki, Estimation of spatial variability of lignite mine dumping ground soil properties using CPTu results, Studia Geotechnica et Mechanica, 38(1), 3-13 (2016)

15. S.K. Jha, J. Ching, Simulating Spatial Averages of Stationary Random Field Using the Fourier Series Method, Journal of Engineering Mechanics, 139, 594-605 (2012)

16. D.V. Griffiths, P.A. Lane, Slope stability analysis by finite elements, Geotechnique, 49 (3), 387-403 (1999)

17. K. Gorska, Z. Muszyński, J. Rybak, Displacement monitoring and sensitivity analysis in the observational method, Studia Geotechnica et Mechanica, 35 (3), 25-43 (2013) 